\title{
What is Capability for Suicide? A Review of the Current Evidence
}

Austin J. Gallyer, M.S. ${ }^{1}$, Greg Hajcak, Ph.D. ${ }^{1,2}$, \& Thomas E. Joiner, Ph.D. ${ }^{1}$

${ }^{1}$ Department of Psychology, Florida State University

${ }^{2}$ Department of Biomedical Sciences, Florida State University

\section{Author Note}

This manuscript is available as a preprint: https://psyarxiv.com/xgwa5/. This work was supported by a grant from the National Institute of Mental Health (T32MH093311). This work was also supported in part by the Military Suicide Research Consortium (MSRC), an effort supported by the Assistant Secretary of Defense for Health Affairs under Award No. (W81XWH-16-2-0004). Opinions, interpretations, conclusions, and recommendations are those of the author and are not necessarily endorsed by the MSRC or the Department of Defense.

Correspondence concerning this article should be addressed to Austin J. Gallyer, M.S., 1107 West Call St., Tallahassee, FL, 32306. Email: agallyer@neuro.fsu.edu. 


\begin{abstract}
It is well known that most people who think about suicide do not die by suicide. Capability for suicide, a construct proposed by Joiner (2005) within the interpersonal theory of suicide, was relatively novel in that it explained a potential mechanism by which individuals move from thinking about suicide to engaging in suicidal behavior. In this paper, we examine Joiner's original conceptualization of the nature of capability for suicide, and review the evidence for and against this conceptualization. We examine the evidence for specific constructs that comprise capability for suicide, how capability for suicide develops, whether it can change, and possibilities regarding the functional neural correlates of capability for suicide. Finally, we discuss issues in measuring capability for suicide, and make recommendations for the future study of capability for suicide.
\end{abstract}

Keywords: suicide, capability for suicide, interpersonal theory 


\section{What is Capability for Suicide? A Review of the Current Evidence}

Suicide is a worldwide public health concern that has spurred decades of research aiming to predict and ultimately prevent suicide (Franklin et al., 2017). Every year, over 800,000 people die by suicide worldwide (Naghavi, 2019). While this figure is staggering (and likely an under-estimate), approximately 160 million have suicidal thoughts (World Health Organization, 2014). Consistent with these data, it is well-established that most who think about suicide do not die by suicide (Fazel \& Runeson, 2020). A natural follow-up question is: why do most who think about suicide choose not to die by suicide? This question was central to the development of one of the most empirically scrutinized contemporary theories of suicide: the interpersonal theory of suicide (ITS; Joiner, 2005).

Briefly, the ITS proposes that desire for suicide occurs only when people experience both the feeling that they do not belong (i.e., thwarted belongingness), and perceive that they are a burden on others and/or that self-hood itself is a burden (i.e., perceived burdensomeness). The theory is explicit that these states are most pernicious when they are viewed as intractable. The ITS further hypothesizes that regardless of an individual's desire for suicide, they will not engage in suicidal behavior unless they also have the capability for suicide. This construct, described originally as the acquired capability for suicide, was proposed to consist of physical pain tolerance and fearlessness about death (Joiner, 2005). The necessity of these constructs for engaging in suicidal behavior stems from the idea that suicide is difficult to engage in because it is directly opposed to the instinct of selfpreservation.

The ITS was relatively novel in that it was the first theory to propose a construct that differentiates those who think about suicide from those who ultimately attempt and die by suicide. That is, it was the first theory of suicide that functioned within an ideation-to-action 
framework (Klonsky et al., 2018). Though research is ongoing, a recent meta-analysis suggests that capability for suicide, when present in the context of elevated thwarted belongingness and perceived burdensomeness, is related to suicide attempt history (Chu et al., 2017). This evidence, along with laboratory experimental evidence, suggests that capability for suicide may be an important factor for the transition from suicidal thoughts to engaging in suicidal behavior (e.g., Hames et al., 2017).

Whether capability for suicide is necessary for suicidal behavior to occur is an important area for study. Just as important, however, is determining what capability for suicide is. Therefore, rather than focus on whether capability for suicide is related to suicidal behavior, the purpose of this review is to examine the current evidence for what capability for suicide is ${ }^{1}$. We do this by exploring whether the evidence supports capability for suicide as it was originally proposed by Joiner (2005), and further elaborated on by Van Orden and colleagues (2010). Specifically, this review will focus on these questions: (1) Which constructs compose capability for suicide?; (2) How does capability for suicide develop?; (3) Can capability for suicide change?; and (4) What are the functional neural correlates of capability for suicide? We also discuss how capability for suicide has been measured over time. Last, we then end this paper by reviewing remaining questions about capability for suicide, and propose future research directions.

\section{Which Constructs Compose Capability for Suicide?}

\section{Original Hypothesis}

The ITS originally conceptualized capability for suicide as consisting of two primary constructs: fearlessness about death and elevated pain tolerance (Joiner, 2005). These two constructs were thought to be correlated with one another to form a higher order construct (i.e., capability for suicide) — and it was proposed that a person could enact lethal or near-

1 For our methods for finding and selecting articles, please see Supplementary Material 1. 
lethal self-harm in the context of serious desire for suicide only if both fearlessness about death and elevated pain tolerance were also present. As we discuss in the next section, in recent years, the concept of capability for suicide has been elaborated. This development was intimated by Joiner (2005): “...[suicide] is impossible to do without previous experiences that allay the fear of self-injury, inure people from the pain of self-injury, and build knowledge that facilitates self-injury [emphasis added]." The knowledge needed to enact self-injury and the development of other constructs illustrate that the construct of capability for suicide expanded beyond what was emphasized in the original conceptualization of the ITS.

\section{Current Evidence}

Work since the development of the ITS confirms that fearlessness about death and physical pain tolerance are related. In a study validating an English self-report scale of fearlessness about death, self-reported fearlessness about death was related to behavioral measures of physical pain tolerance (Ribeiro et al., 2014). In a study that examined a German self-report measure of both physical pain tolerance and fearlessness about death, a two-factor solution was the best fit, with the pain tolerance and fearlessness about death factors demonstrating a moderate correlation ( $r=.34$; Wachtel et al., 2014). A two-factor solution was also found in a Chinese version of the scale, with fearlessness about death and pain tolerance showing a a similar moderate correlation ( $r=.34$; Yang et al., 2019). Though the measurement models in both of these studies demonstrated acceptable fit, neither study tested alternative models. Moreover, the measurement models of these studies suggest that fearlessness about death and physical pain tolerance are separate, correlated factors, but that they do not then load onto a higher order factor (i.e., capability for suicide) as described by the ITS.

Klonsky and May (2015) introduced an ideation-to-action theory of suicide called the three-step theory of suicide (3ST). Like the ITS, the 3ST focuses prominently on capability 
for suicide. In the 3ST, Klonsky and May (2015) proposed that capability for suicide consists of three components: dispositional, acquired, and practical capability for suicide. The first two components, dispositional and acquired, emphasize points we will discuss in more depth later in our review (i.e., whether capability for suicide has a genetic component, as well as an environmental component that is acquired over time). Practical capability for suicide is described as the knowledge of, and access to, means for suicide. The important role of access to lethal means in relation to suicide risk is supported by studies which have shown that firearm legislation, particularly more strict firearm legislation, decreases the rate of suicides using firearms, and has no effect on the suicide rate using other means ${ }^{2}$ (Anestis et al., 2019; Boor \& Bair, 1990; Fleegler et al., 2013; Ludwig \& Cook, 2000). Conceptually, the question is whether practical capability correlates with other components of capability for suicide, namely, fearlessness about death and physical pain tolerance. For example, a person that is high in fearlessness about death may also be more likely to keep firearms loaded and unlocked. Moreover, a person who keeps their firearm more easily accessible, or fires more frequently, may develop higher fearlessness about death over time.

One of the first studies to begin to address whether practical capability is related to other components of capability for suicide was conducted among a sample of United States (U.S.) military personnel (94.5\% National Guard; Khazem et al., 2015). The authors found that fearlessness about death was higher in soldiers who kept their firearms unsecured and loaded compared to soldiers who kept their firearms secured and/or unloaded. Another study conducted among a community sample found that more lifetime experiences with firing a firearm were associated with increased fearlessness about death and physical pain tolerance

2 This latter point is important to note as it provides evidence that when individuals are not given access to their preferred method of suicide, they usually do not engage in means substitution (i.e., usually do not use a different method). 
(Anestis \& Capron, 2017). A similar finding emerged in a separate study that focused on a community sample, and expanded past results to show that unsafe firearm storage was also associated with capability for suicide (Butterworth et al., 2018). Finally, in a large sample of National Guard service-members, Goldberg and colleagues (2019) found that increased firearm ownership and familiarity were associated with increased self-reported capability for suicide (i.e., a measure with items that measured both fearlessness about death and pain tolerance). Interestingly, when examining their sample grouped by reasons for owning the firearm, owning a firearm for self-protection and owning a military weapon were associated with increased self-reported capability for suicide, while owning a weapon for a hobby was not associated with capability for suicide. Finally, one study conducted an exploratory factor analysis with twenty-seven items derived from several scales purported to measure different aspects of capability for suicide (Shahnaz et al., 2020). They found a three-factor solution, with factors that they labeled as practical capability, fearlessness about death, and pain tolerance. Similar to factor analysis studies that did not examine practical capability, they found that these three factors were weakly correlated with each other (i.e., $r=0.15-0.35$ ).

While this set of studies provides strong evidence that the elements of practical capability are associated with fearlessness about death and physical pain tolerance, given the cross-sectional nature and other design characteristics, these studies are unable to answer whether practical capability and the other components of capability for suicide arise from the same latent factor (i.e., that they are "the same thing"). Rather, it could be argued that each of the components of practical capability (i.e., access to and knowledge of lethal means) are painful and provocative events that lead to increased fearlessness about death. Moreover, it is also plausible that those who have higher fearlessness about death and/or physical pain tolerance are more likely to gravitate to firearms and store them less safely. Therefore, further studies are needed to better answer whether practical capability, fearlessness about death, and 
physical pain tolerance, index the same construct.

Though practical capability is perhaps the most well-supported construct other than fearlessness about death and physical pain tolerance, other candidates as components of capability for suicide are worth noting. For example, a more specific version of fearlessness about death is fearlessness about suicide. In a sample of psychiatric inpatients with a history of multiple suicide attempts, fearlessness about suicide was found to be related to the number of suicide methods used and the number of violent methods used, whereas fearlessness about death was not related to these outcomes (Gauthier et al., 2018). However, this study was limited by using single-item assessments of fearlessness of suicide and death.

Another possibility is that interoceptive deficits comprise suicide capability. Interoception is a multidimensional construct that represents a person's ability to sense, accept, and respond to internal sensations and emotions (Craig, 2003; Mehling et al., 2012). Recent work has found that one component of interoception, called body trust, may have a particularly strong relationship to suicidal behavior compared to other facets of interoception (Duffy et al., 2019; Rogers et al., 2018). Low body trust is thought to lead to detachment from the body, and this detachment can lead to self-destructive behavior (Orbach, 1996). However, much of this work has been cross-sectional and has not directly examined the relationship between interoceptive deficits and the components of capability for suicide. Because of these limitations, interoceptive deficits - and body trust in particular - may increase fearlessness about death and pain tolerance. Detachment from the body, in particular, may increase physical pain tolerance. Therefore, research is still needed, particularly in longitudinal or experimental designs, to determine how interoceptive deficits relate to capability for suicide.

In summary, though there is strong evidence that physical pain tolerance and fearlessness about death are correlated, whether they load onto a single construct is an open 
question. Moreover, practical capability for suicide, though alluded to by Joiner (2005), is perhaps the most supported construct that was not emphasized in the original conceptualization of capability for suicide that was brought to the forefront by Klonsky and May’s 3ST (2015). Despite this support for practical capability, the current evidence suggests that much work is still needed to determine whether fearlessness about death, physical pain tolerance, and practical capability "hang together" as a single construct. It could be argued that the evidence so far showing only moderate correlations between different facets of capability for suicide is to be expected, and that capability for suicide should not be thought of as a higher order construct, but rather as a term to describe constructs that are necessary for suicide attempts to occur. We think this is problematic, and that in order for capability for suicide to be considered its own construct, the presence of a higher-order factor is imperative. Specifically, we reason that defining capability for suicide as constructs that are necessary for suicide to occur, but that don't necessarily correlate highly or load onto a higher order factor, would be akin to defining a psychiatric disorder based on symptoms that do not correlate and do not load onto a higher order construct. If stronger evidence is found that does not support capability for suicide as a higher order construct, then it may be prudent to consider other terms, as capability for suicide intimates a single construct. Finally, besides practical capability, other constructs also deserve further investigation, including fearlessness about suicide and interoception.

\section{How Does Capability for Suicide Develop?}

\section{Original Hypothesis}

As originally described, the ITS proposed that capability for suicide is acquired largely through painful and provocative events (Joiner, 2005; Van Orden et al., 2010) ${ }^{3}$. Events

\footnotetext{
3 The roles of factors like familiarity with suicide method and genetics as aspects of capacity were also noted in the 2005 work, though were not emphasized to the same degree as were fearlessness about death and physical pain tolerance.
} 
such as past suicidal behavior, physical injuries, childhood abuse (particularly when physical in nature), active combat, and contact sports, were described as potential events leading to the development of capability for suicide, particularly when these experiences are direct and frequent or repeatedly experienced. The basis for this conceptualization derived primarily from opponent-process theory. Briefly, opponent-process theory suggests that with repeated exposure to an affective stimulus, the reaction to that stimulus shifts over time such that the stimulus no longer elicits the original response, and instead, an opposite response emerges and that is strengthened over repetitions (Solomon \& Corbit, 1974). Thus, exposure to events such as self-harm and active combat were hypothesized to lead to higher pain tolerance and increased fearlessness about death, and in turn, make engaging in suicidal behavior more feasible under conditions in which it is desired. That capability for suicide develops through an opponent process rather than only habituation is an important distinction to make. For example, one possibility is that habituation through painful and provocative events makes attempting suicide a more emotionally neutral or fearless behavior. However, the ITS proposed that in addition to making suicide a more neutral behavior, that an attraction to suicide may occur.

Perhaps the strongest evidence for this conceptualization of the development of capability for suicide at the time was that history of suicide attempts is, relative to most other risk factors, a uniquely strong predictor of future suicidal behavior, including dying by suicide (Brown et al., 2000; Joiner et al., 2005). Through the lens of the ITS, this relationship is present because the most direct route for obtaining the physical pain tolerance and fearlessness about death required to engage in suicidal behavior is by engaging in past suicidal behavior. A related line of evidence had also shown that the number of previous suicide attempts is associated with greater medical damage from the most serious suicide attempt (Soloff et al., 2000; Soloff et al., 1994). Previous research had also shown that even 
relatively minor painful events are associated with increased risk for suicide or history of suicidal behavior. For example, one study found that those who had died by suicide were more likely to have tattoos compared to case-matched controls who had died from an accident (Dhossche et al., 2000). Another study found that women who engage in frequent self-harm had more surgeries than controls (Rosenthal et al., 1972). Other associations also supported the possibility that events that may inure individuals to death can lead to increased suicide risk. For example, research had shown that physicians are at higher risk for dying by suicide than those in the general population (Schernhammer \& Colditz, 2004).

In summary, the ITS, as originally proposed by Joiner (2005) forwarded that capability for suicide - comprised of fearlessness about death and elevated physical pain tolerance-largely developed through exposure to painful and provocative events (but see Footnote 2). This proposal was supported by several lines of evidence, most notably that previous suicidal behavior is strongly associated with future suicidal behavior (Joiner et al., 2005). Since the ITS was proposed, however, this hypothesis has been more directly tested.

\section{Current Evidence}

There have been a number of cross-sectional studies that have found a relationship between history of painful and provocative events and capability for suicide across a variety of samples, including undergraduate students, psychiatric outpatients, U.S. firefighters, community adults, and prison inmates (Anestis et al., 2011, 2014; Anestis \& Joiner, 2012; Bauer, Gai, Joiner, et al., 2020; Bender et al., 2011, 2012; Franklin et al., 2011; Gauthier et al., 2014; Granato et al., 2015; Hawkins et al., 2014; Mitchell, Cukrowicz, et al., 2015; Mitchell et al., 2014; Mitchell, Jahn, et al., 2015; Smith et al., 2013; Stanley, Hom, Gallyer, et al., 2019; Teismann et al., 2015; Van Orden et al., 2008). In contrast, there have been other crosssectional studies that have not found this relationship with various measures of painful and provocative events (Bauer et al., 2018; Butterworth et al., 2017; Ribeiro et al., 2014). In 
perhaps one of the strongest cross-sectional tests of the relationship between painful and provocative events and capability for suicide, Bauer and colleagues (2020) used a large dataset from the Military Suicide Research Consortium with participants in the U.S. military, U.S. military veterans, and U.S. civilians with no history of military service to examine whether fearlessness about death differs between those with a previous suicide attempt who used varying methods to make their attempt. According to the ITS, more lethal methods of suicide require greater capability for suicide (Joiner, 2005; Van Orden et al., 2010). However, Bauer and colleagues did not find differences in fearlessness about death as a function of suicide method. In fact, they were able to find confirmatory evidence that these groups did not differ in fearlessness about death by using an analysis called equivalence testing (Lakens, 2017).

If the hypothesis that capability for suicide is largely acquired over time through exposure to painful and provocative events is true, then studies using longitudinal designs should find that capability for suicide does not change, unless painful and/or provocative events occur; conversely, for those who have high capability for suicide, it should slowly decrease over long periods of time if painful and/or provocative events do not occur or occur only infrequently. Two longitudinal studies provide mixed results regarding whether capability for suicide is increased by a particularly strong form of painful and provocative event: non-suicidal self-injury (NSSI). A study conducted in a large sample of undergraduate students found that frequency of NSSI predicted self-reported capability for suicide one year later (Willoughby et al., 2015). In contrast, a study by Koenig and colleagues (2017) did not find a relationship between NSSI and pain tolerance measured by a cold pressor task. Based on the results of these studies, it is tempting to conclude that perhaps painful and provocative events change fearlessness about death, but not pain tolerance. However, all three of these studies have limitations. The study by Koenig and colleagues (2017) had a small sample (i.e., 
$N=37$ ), thus possibly limiting statistical power to detect effects. On the other hand, the study by Willoughby and colleagues (2015) used cross-panel autoregressive modeling. This model has been found to be problematic because it assumes that individuals vary over time around the same mean and standard deviation, and that there are no trait-like individual differences (Hamaker et al., 2015). This can cause the parameters to be biased leading to inappropriate causal inferences (Hamaker et al., 2015). Moreover, rather than being identified, this model is only "just identified" with two waves of data, as used by Willoughby and colleagues (2015). Because of this, it is impossible to assess whether the data fit the model or whether other models would be more appropriate because just identified models will always fit the data perfectly. Therefore, longitudinal studies with large samples and multiple assessment points are needed to examine whether NSSI, or any other painful and provocative experience, change capability for suicide. Specifically, if change in capability for suicide is determined by the frequency of painful and provocative experiences, such that for those with high capability for suicide, their capability for suicide should remain stable if painful and provocative events continue, and slowly decrease over time if painful and provocative events stop. Conversely, for those low in capability for suicide, capability for suicide should remain stable and increase in the presence of painful and provocative events, albeit depending on the frequency and severity of the experiences.

Bryan and colleagues (2016) found evidence that may be at odds with this hypothesis. They tested military personnel's capability for suicide at several time points starting at the beginning of training before deployment, and ending 12 months after deployment to Iraq, and found that capability for suicide remained stable across the study, even among those who reported frequent combat exposure while deployed. This suggests that even among those who experienced significant painful and provocative events, their capability for suicide did not increase as expected. A limitation of this study is that those with greater capability for suicide 
may be more likely to enlist in the military in general, and for combat roles in particular. Thus, Bryan and colleagues may not have seen an increase in capability for suicide due to a ceiling effect. Moreover, a study by Ribeiro et al. (2020) may provide confirmatory evidence that nonfatal suicide attempts increases fearlessness about death. They collected a large sample of adults recruited from self-injury, suicide, and mental health web forums. Their sample was relatively high risk, with most reporting a history of self-injury, nearly half engaging in self-cutting in the month before the baseline assessment, and over half had a previous suicide attempt. In this study, they measured the participant's fearlessness about death and self-injurious thoughts and behaviors at baseline. They then conducted follow-up assessments three, fourteen, and twenty-eight days post-baseline. At each assessment they measured fearlessness about death, and any self-injurious behaviors since baseline. They then conducted multiple mediation models to examine whether baseline fearlessness about death had indirect effects on follow-up (at three, fourteen, and twenty-eight days post-baseline) fearlessness about death through non-suicidal self-cutting, aborted suicide attempts, interrupted suicide attempts, and nonfatal suicide attempts. They failed to find any significant indirect effects between baseline fearlessness about death and follow-up fearlessness about death. Moreover, they failed to find a significant direct effect between baseline fearlessness about death and nonfatal suicide attempts between baseline and follow-up. However, they did find direct effects between nonfatal suicide attempts and follow-up fearlessness about death. Though they pointed to their failed indirect effects as evidence against capability for suicide being changed over time, it is our view that the indirect effects were not the most important effects in their model. Their indirect effects tested whether baseline fearlessness about death has an indirect effect on follow-up fearlessness about death through a number of selfinjurious behaviors, but capability for suicide is not theorized to have a direct effect on selfinjurious behaviors. Rather, capability for suicide is theorized to be a moderator between 
other constructs that cause suicidal desire. Instead of their indirect effects, our view is that their direct effect between nonfatal suicide attempts and follow-up fearlessness about death while controlling for baseline fearlessness about death is strong evidence that fearlessness about death may be increased through particularly salient painful and provocative events-in this case, a nonfatal suicide attempt. Importantly, in contrast to Bryan and colleagues (2016) paper, Ribeiro and colleagues (2020) reported that their variables were normally distributed, and therefore did not suffer from restriction of range that may explain Bryan and colleagues' null effects.

Based on this mixed evidence, questions remain whether capability for suicide develops largely or only from experiencing painful and provocative events or not. How, then, does capability develop? To address this question, Smith and colleagues (2012) conducted a behavioral genetics study using twin data from the Minnesota Twin Family Study to determine the estimated environmental and genetic effects on capability for suicide. They found that a significant portion of capability for suicide is determined by non-additive genetic effects ( $47 \%$ of variance, $95 \% \mathrm{CI}=30$ to $60 \%$ ), with the rest determined by non-shared environmental effects, which may be explained by engaging in, or exposure to, painful and provocative events. In comparison, the other two components of the ITS, thwarted belongingness and perceived burdensomeness, were estimated to be determined entirely by various environmental effects. This study did have significant limitations, including the use of proxy measures for each of the ITS's constructs that demonstrated poor internal consistency. Moreover, this study was conducted in an entirely male sample that was almost exclusively White, limiting the generalizability of the findings.

Based on the results of these studies, the variance in capability for suicide appears to have an even stronger genetic component than was originally conceptualized by the ITS (Joiner, 2005). Which gene or genes contribute to the development of capability for suicide 
remains an open question that we will discuss in the final section of our review. These results also show that though capability for suicide appears to have a strong environmental component, the role of painful and provocative events is less clear, with studies showing contradictory findings. One explanation for these findings is the possibility that, at least for some, painful and provocative events, but particularly suicide attempts, can have a sensitizing effect (Stanley et al., 2018). That is, in some people suicide attempts may reduce the fear associated with a suicide attempt, while for others, it may be a terrifying, even traumatic experience. This possibility has been supported by work showing evidence that some suicide attempt survivors experience suicide attempt-related posttraumatic stress disorder (Bill et al., 2012; Stanley et al., 2019). Thus, there may be individual differences in for whom painful and provocative events have a habituating effect, and for whom it has a sensitization effect, based on the complicated interplay between an individual's genetic risk and experience from painful and provocative events.

\section{Can Capability for Suicide Change?}

\section{Original Hypothesis}

Capability for suicide was originally hypothesized to increase over the course of months, or even years, of exposure to painful and provocative events ${ }^{4}$, or, in the case of those with high capability, decrease over long periods when painful and provocative events were not present (Joiner, 2005). Indeed, though capability for suicide is hypothesized to be the necessary factor for individuals who have serious desire to die by suicide to engage in suicidal behavior, Joiner (2005) warned that capability for suicide, “...may not be a useful target for [suicide] prevention efforts, because this quality is relatively static and not very malleable, at least not over the short-term."

The reasoning for this conceptualization was largely drawn from how capability for

4 Though this does depend on the frequency and severity of painful and provocative events. 
suicide was thought to develop — that is, through repeated exposure to painful and provocative events. Thus, it was thought that reversing this process, on average, would take as much time and effort as it took to develop capability for suicide (but see footnote 3). As we just reviewed, capability for suicide has a large genetic component and the role of painful and provocative events is less clear, with the possibility that some individuals habituate whereas others become sensitized. This leads to the question of whether capability for suicide remains relatively stable in the short-term, as originally proposed, or whether it may change more rapidly.

\section{Current Evidence}

Zuromski and colleagues (2018) found evidence that, at least for some, capability for suicide can change in a relatively short time-frame. They recruited a large sample of undergraduate students and assessed their fearlessness about death using a self-report scale every three days over a fifteen-day period. They then used latent class growth modeling, a statistical technique that is used to try to identify groups with different trajectories of a construct over time. Using this technique, they identified three classes: (1) participants whose fearlessness about death started relatively high, and continued to increase over the course of the study (20.80\% of the sample); (2) a group whose fearlessness about death was average, and did not change over the course of the study (66.20\% of the sample), and; (3) a group whose fearlessness about death was low, and decreased over time (13.00\% of the sample). This suggests that, at least for some, capability for suicide can change in a relatively short time (i.e., fifteen days).

A similar study examined a sample of patients presenting to a residential eating disorder treatment facility to identify groups with different trajectories of capability for suicide over the course of the study (Velkoff \& Smith, 2019). Using latent class growth modeling, they found that all participants were best allocated to one group whose capability 
remained stable throughout the course of the study. A key difference in this study compared to the study by Zuromski and colleagues (2018) is that their study was conducted over the course of eight weeks, rather than fifteen days. Together, these studies suggest that when capability for suicide is observed in longer intervals, it remains stable, but when it is observed over shorter intervals, it fluctuates for some participants. Thus, if participant's capability for suicide is measured in much shorter intervals (e.g., several times per day), it may reveal greater short-term variability.

Recent developments in technology have made it possible to better assess short-term fluctuations in constructs by sending self-report questions to participants' phones multiple times per day. This method, often called ecological momentary assessment (EMA; Shiffman et al., 2008; Stone \& Shiffman, 1994), has been used to study constructs like suicidal ideation, hopelessness, perceived burdensomeness, and thwarted belongingness, and has generally found that these constructs can change dramatically over the course of a few hours (Hallensleben et al., 2018, 2019; Kleiman et al., 2017). EMA was used by Spangenberg and colleagues (2019) to examine whether capability for suicide demonstrates large fluctuations over short time intervals. They found that about half of the variance of fearlessness about death and perceived pain tolerance was due to within-person variability. Indeed, the intraclass correlation coefficients (ICCs) of capability for suicide were similar to those seen when measuring suicidal ideation, thwarted belongingness and perceived burdensomeness (Kleiman et al., 2017). Though these studies measured their respective constructs at slightly different frequencies, making the ICCs not necessarily directly comparable, the study by Spagenberg and colleagues supports the notion that capability for suicide can change over the course of a day.

Given that capability for suicide may change in the short-term, it is of interest that only four studies have been published attempting to experimentally manipulate capability for 
suicide. The first study recruited undergraduate students and had them either play a racing video game, or a first-person shooter game (Teismann et al., 2014). They found that those who played the first-person shooter had higher physical pain tolerance than the control group. The second study, conducted by Franklin and colleagues (2016), used an app that leveraged evaluative conditioning to increase aversion to suicidal stimuli. They found that those in the active group showed a significant increase (i.e., $d=.50$ ) in implicitly measured aversion to suicidal stimuli, compared to those in the control group. Though this implicit measure was not necessarily designed as a measure of capability for suicide, this study provides some evidence that capability for suicide can be reduced. The third study used acetaminophen to examine whether it would increase capability for suicide, and an experimental reading task to examine whether it would decrease capability for suicide (Chiurliza \& Joiner, 2018). Interestingly, acetaminophen had no effect on physical pain tolerance or fearlessness about death, and the reading task had a significant effect in the opposite direction by increasing physical pain tolerance. The fourth study, though not designed to examine capability for suicide, examined whether what has been called "power posing" (i.e., physical poses that were argued to be more assertive/aggressive) has an effect on pain tolerance. Though the authors found that having participants engage in "power poses" significantly increased physical pain tolerance (Bohns \& Wiltermuth, 2012), it should be noted that power posing is a concept that has been fraught with replication problems, with the lead author of the original study concluding that there is no power posing effect (Carney, n.d.).

Together, these findings provide weak evidence that capability for suicide can be experimentally manipulated, and in the case of one study, the direction of the effect was counter-intuitive to what would be expected ${ }^{5}$. We note a disconnect between EMA studies

5 We want to emphasize that we conclude there is weak evidence for social or cognitive effects on capability for suicide. This excludes pharmacological interventions, such as morphine, which clearly have a strong effect on pain tolerance. 
and experimental studies: EMA studies show that capability for suicide can fluctuate in the short-term (i.e., a day later), whereas experimental studies have, at best, provided very weak or preliminary evidence supporting the possibility that capability for suicide can be changed by environmental conditions.

There are at least two possibilities for this discrepancy. First, perhaps capability for suicide can only be changed through very powerful manipulations that have yet to be tested. For example, the four studies to date have used subtle manipulations that attempted to manipulate capability for suicide that were likely to go unnoticed by the participant. Stronger, more noticeable manipulations may be needed to induce temporary changes in capability for suicide. A second possibility is that capability for suicide fluctuates, but that it fluctuates in the short-term largely independent of the environment. Rather, perhaps the average level of capability for suicide is determined by the long-term accumulation (or long-term absence) of painful and provocative events, but the temporal variability of capability for suicide may be determined by genetic effects. This, however, remains to be tested.

To summarize, capability for suicide appears to change when assessments are close in time, though it remains to be seen whether the frequency of assessment is having an effect on capability for suicide. Indeed, research using the Beck Depression Inventory-ii (BDI-ii; Beck et al., 1996) found that randomizing the frequency of administering the BDI-ii had an effect on the average score over time (Longwell \& Truax, 2005). Therefore, it is possible that capability for suicide shows a similar pattern. That is, capability for suicide may not truly change in the short-term, but remain stable in the long-term; shorter-term effects may be the result of measurement frequency itself. Assuming that assessment effects are absent or negligible, efforts to influence capability for suicide in the short-term have, at best, provided weak evidence that it can be manipulated. In contrast to EMA studies, when viewed over long periods, capability for suicide appears to remain relatively stable. This is consistent with 
work by Bryan and Rudd (2016), in which they characterize suicide risk as a temporally dynamic interplay between various risk factors, which can cause changes in the individual experiencing suicidal symptoms, and in one another. Though this short description does not fully characterize their view, the important component to illustrate here is that some risk factors are thought to have a set point, and, when changed, tend to return to their set point. Therefore, each person's capability for suicide may have a set-point determined by the longterm accumulation of painful and provocative events; both the degree to which capability can fluctuate, and how quickly it returns to baseline, may be genetically determined. This approach to capability for suicide remains to be utilized, though early research using dynamic systems modeling has shown that capability for suicide may play an important role in the dynamics of suicide risk (Rogers \& Joiner, 2019).

\section{What are the Functional Neural Correlates of Capability for Suicide?}

\section{Original Hypothesis}

Though Joiner (2005) discussed the evidence for the neurobiology of suicide at length (i.e., an entire chapter dedicated to the topic), how capability for suicide is represented in neurobiology, in particular, was not discussed. Rather, the role of serotonergic dysfunction, and how this may lead to impulsivity resulting in engaging in behaviors that may increase capability for suicide was emphasized (Joiner, 2005; Van Orden et al., 2010). Perhaps as a consequence, little-to-no research on the functional neural correlates of capability for suicide was conducted for many years. However, in recent years, this area has begun to accumulate evidence, providing clues to the potential functional neural correlates of capability for suicide.

\section{Current Evidence}

To date, no study has directly examined functional neural correlates of capability for suicide. One study that indirectly examined the functional neural correlates of capability using fMRI was a meta-analysis which used proxy constructs, such as "fear" and "pain 
processing," stratifying their results by sex (Deshpande et al., 2016). Some brain regions that were activated in relation to these constructs across males and females included the anterior cingulate cortex, the insula, putamen, and claustrum. Though more fine-grained spatial information is needed, these areas suggest that capability for suicide is broadly related to neural processes involved in cognitive, emotion, and pain processing. Other studies, though not conducted with capability for suicide in mind, have examined the functional neural correlates of pain. This work suggests that the anterior cingulate cortex, somatosensory cortex, and the insula may be integral to the subjective experience of pain (Coghill et al., 2003; Starr et al., 2009). In contrast to the work using fMRI to examine capability for suicide, there is a larger body of evidence using event-related potentials (ERPs) to investigate the neural correlates of capability for suicide.

Briefly, ERPs are direct measures of neural activity that are derived from the electroencephalographic (EEG) time-locked to specific, repeated events (Luck, 2014). ERPs are calculated by averaging the electrocortical response to repeated trials of a specific task event (e.g., error commission, receipt of a reward). ERPs have numerous strengths, including high temporal resolution, and lower cost relative to fMRI data. Importantly, ERPs have been shown to have potential clinical utility for various forms of psychopathology, such as depression and anxiety (Hajcak et al., 2019). An ERP that has shown to have relevance to capability for suicide is the late positive potential (LPP). The LPP is an ERP evident as a sustained positive deflection in response to viewing emotionally-valenced content (Cuthbert et al., 2000; Weinberg \& Hajcak, 2011). Variability in the amplitude of the LPP is related to the motivational salience of the stimulus, with the LPP exhibiting the greatest amplitudes when in response to stimuli related to survival, such as images of sex and mutilation (Weinberg \& Hajcak, 2010).

In a relatively large sample of psychiatric outpatients, Weinberg and colleagues (2017) 
found that suicide attempt survivors had a blunted LPP to threatening images compared to controls with suicidal ideation, but without a previous suicide attempt. A similar study was conducted by Albanese and colleagues (2019) that included three conditions: passive viewing, where participants were given no instructions; a decrease condition, where participants were instructed to decrease their emotional response; and an increase condition, where participants were told to increase their emotional response to the stimuli. Albanese et al. (2019) showed that suicide attempt survivors with multiple previous suicide attempts had a significantly reduced response to threat and mutilation images during the emotion decrease condition. Together, these two studies suggest that suicide attempt survivors' neural reactivity to threat is diminished, which is in line with what would be expected for those high in fearlessness about death.

Another recent study examined whether the LPP to threat was associated with fearlessness about death directly (Bauer, Albanese, Macatee, et al., 2020). In this study, the authors recruited a community sample $(N=280)$ with the majority of participants having some form of psychopathology $(90.1 \%)$. They found that higher fearlessness about death was associated with a diminished LPP to images of threat/mutilation when controlling for depression and state anxiety, but only when they limited their sample to participants with suicidal ideation, significantly decreasing their sample size $(n=62)$. Because the authors conducted these regressions in two separate analyses, rather than including suicidal ideation as a moderator, it is unknown whether the difference between the non-significant association in the full sample, and the significant association in the suicidal ideation sample, is statistically different. Nevertheless, these results suggest that increased fearlessness about death is associated with a blunted LPP to threatening images. These studies provide evidence that the neural systems that give rise to the LPP are related to capability for suicide. Given that capability for suicide has been shown to be related to a diminished LPP to threatening 
images and not rewarding images as well is particularly notable. Specifically, depression is often characterized by a blunted LPP to both threatening and rewarding stimuli (Klawohn et al., 2020; Weinberg et al., 2016). If capability for suicide is only associated with a blunted LPP to threatening images and not rewarding stimuli, and this effect is large enough, this may make the LPP to threatening stimuli a biomarker for those at risk for suicidal behavior. What then do these studies say about the possible functional neural correlates of the LPP and capability for suicide? There are several possibilities, but the two we will focus on here include the locus coeruleus (LC) and the amygdala.

First, evidence suggests that the LPP is linked to a protracted orienting response, evident as a sustained P300 (Hajcak \& Foti, 2020). Hajcak and Foti suggest that this orienting response is reflected in the LPP/P300, and may result from phasic activity of the LC norepinephrine system. This is supported by work showing that activity in the LC is related to other measures of the orienting response (Nieuwenhuis et al., 2011; Sara \& Bouret, 2012).

In addition to the LC, research has also found a relationship between LPP amplitude and activation in the amygdala (Liu et al., 2012). In line with the work showing that the LPP is related to orienting to relevant stimuli, the amygdala has also been shown to have a role in the detection of threat and other salient stimuli (Öhman, 2005). Based on this, the amygdala and LC may index the same coordinated neural response to salient stimuli. Indeed, there is some evidence that threat learning in the amygdala is modulated by the orexin/hypocretin neuropeptide system through the LC (Sears et al., 2013). Moreover, there is evidence that these neuropeptides are involved in fear extinction (Flores et al., 2014). Research also suggests that at least parts of fear extinction are encoded in medial prefrontal cortexamygdala circuits (Cho et al., 2013). Finally, one study found evidence that those with MDD, who most died by suicide (i.e., 13 out of 15 ), had fewer norepinephrine transporters in the LC compared to controls (Klimek et al., 1997). 
The evidence we have reviewed suggests that capability for suicide, but particularly fearlessness about death, may involve several neural circuits, including pathways between the medial prefrontal cortex, LC, and amygdala. Moreover, the orexin/hypocretin neuropeptide system may also play a role. However, because of the absence of functional neuroimaging studies investigating capability for suicide directly, these ideas remain to be tested. Indirect studies of the functional neural correlates of capability for suicide also point to other brain regions involved in emotion, cognitive, and pain processing, including the anterior cingulate cortex and the insula.

\section{Measurement of Capability for Suicide}

Central to all of the literature we have discussed so far is the measurement of capability for suicide. Using measures that have been shown to allow inferences about relations between constructs is imperative in psychological science, and questionable measurement practices like creating measures on the fly or not investigating psychometric properties can limit the validity and replicability of research findings (Clark \& Watson, 2019; Cronbach \& Meehl, 1955; Flake \& Fried, 2020). Thus, it is important to discuss the state of the literature on the measurement of capability for suicide, and what this research says about the literature we have discussed so far about what capability for suicide is. Because it has been the dominant measurement approach in studying capability for suicide, we will focus on self-report scales of capability for suicide, but many of the points we discuss here could apply to behavioral measures as well.

Like much of the psychological literature, scales developed for measuring facets of capability for suicide have not necessarily undergone formal psychometric scrutiny before being implemented. Shortly after the ITS was introduced, the Acquired Capability for Suicide Scale (ACSS), the scale that is most common in this literature, was first published (Van Orden et al., 2008). In this initial publication, the ACSS was found to have sub-optimal 
internal consistency, and was found not to correlate with measures of depression and suicidal ideation. A separate paper written before this study, but that was not published until later, found that the ACSS was correlated with items assessing fear of suicide and courage to kill themselves in the expected direction (Bender et al., 2011). The ACSS originally consisted of twenty items, with the scale intending to measure fearlessness about death, and physical pain tolerance. However, as discussed by Ribeiro and colleagues (2014) the content of the original twenty-item scale did not match up with these constructs. Instead, seven items were designed to assess fearlessness about death, one to assess physical pain tolerance, and the remaining twelve items were designed to assess painful and provocative events. Because of these issues, Ribeiro et al. (2014) reduced the ACSS to just seven items that all assess fearlessness about death, creating the Acquired Capability for Suicide Scale-Fearlessness About Death (ACSSFAD). In addition to finding that the measurement model was a good fit for the ACSS-FAD, Ribeiro et al. (2014) also found that the measure was invariant across gender in three separate samples of young adults. They also found that the ACSS-FAD was correlated with an item assessing fear of suicide, behavioral physical pain tolerance, and uncorrelated with depression and suicidal ideation. The ACSS-FAD also demonstrated good internal consistency, with $\alpha$ 's ranging from 0.77 to 0.83 . Despite this study showing that the original ACSS did not demonstrate content validity, many studies have still continued to use the full or various shortened versions of the scale (e.g.,_Gutierrez et FAD demonstrated good psychometric properties in the samples collected by Ribeiro et al. (2014), recent evidence has cast doubt on the measurement invariance of the ACSS-FAD. In a large dataset consisting of studies funding by the Military Suicide Research Consortium, the ACSS was found to have very poor model fit for the originally hypothesized factor structure (Rogers et al., 2021). Regarding the ACSS-FAD, evidence was found for lack of measurement invariance across suicide attempt history. Moreover, scalar invariance wasn't 
supported for military deployment history, and partial metric and scalar invariance was only partially supported. These results suggest that ACSS-FAD scores should not be compared across heterogeneous groups.

Other, less commonly used measures of capability for suicide have also largely not undergone formal psychometric scrutiny before being used (e.g., the Suicide Capacity Scale; SCS-3; Klonsky \& May, 2015). However, as discussed previously, the ACSS-FAD, SCS-3, and other scales, all appear to load onto appropriate factors with good construct validity and internal reliability (Shahnaz et al., 2020). The psychometric properties of these other capability for suicide scales individually remain to be seen.

In summary, it may be tempting to conclude that given these findings for the ACSS, ACSS-FAD, and SCS-3, that the literature we have discussed so far cannot be trusted. To this, we say that it is important to remember that it is not the scales themselves that are validated. Rather, "One does not validate a test, but only a principle for making inferences" (Cronbach \& Meehl, 1955). Our view is that while the capability for suicide scales need improvement, we do not have the pessimistic view that findings from studies that use them should be thrown out, or are otherwise uninterpretable. However, particularly given that the ACSS-FAD may not have full measurement invariance, caution is warranted in comparing across certain populations.

\section{Summary, Remaining Questions, and Future Directions}

Significant progress has been made in the understanding of the nature of capability for suicide in the 17 years since Joiner (2005) first introduced the construct. Existing research has both supported and contradicted the early conceptualizations of capability for suicide. While determining what a psychological construct is, by nature, never a process that can reach definitive conclusions, these findings nevertheless leave remaining questions that suggest avenues for future studies on capability for suicide. First, capability for suicide 
appears to be driven by much stronger genetic effects than was originally proposed (Joiner, 2005). However, the specific genes implicated in capability for suicide remain unknown. Some have proposed that genes such as the serotonin transporter may play a role in capability for suicide (Smith et al., 2012). Though this gene may play a role, it is important to note that capability for suicide, like all complex traits/behaviors, is likely polygenic, with each gene having a very small effect on the outcome of interest (Harden \& Koellinger, 2020). Because of this small effect, traditional candidate gene studies, where researchers examine one or a few genes in a sample with hundreds or even a couple thousand of participants, are very likely to lead to false positives. Rather than addressing the problem, this problem is compounded by studies that attempt to find gene $\mathrm{X}$ environment interactions. This has been demonstrated recently with candidate genes for depression, with most traditional candidate gene studies that found significant differences in genes such as the serotonin transporter being false positives (Border et al., 2019). Indeed, this work has shown that to detect significant differences in complex traits like depression and capability for suicide as a function of a single gene (or a set of genes), hundreds of thousands of participants would be needed to achieve sufficient statistical power (Border et al., 2019; Harden \& Koellinger, 2020).

Instead of the candidate gene approach, large samples will be needed to conduct genome-wide association studies (GWAS) to identify relevant genes. These genes could then be examined in animal models to examine what kind of effect they have on fearlessness (and relevant neural circuits) in highly controlled settings. Though suicidal behavior per se cannot be modeled in animals, such an approach could significantly improve our understanding of capability for suicide and suicidal behavior (Gould et al., 2017). Moreover, using a GWAS approach allows for techniques such as calculating polygenic risk scores and genomic structural equation modeling, to examine how the genomics of capability for suicide may 
relate to other constructs.

Given that capability for suicide appears to have a genetic and environmental component that may be mediated through painful and provocative events, questions remain regarding when capability for suicide develops. Long-term studies with frequent measurements of capability for suicide with samples at various ages are needed to pinpoint when capability for suicide develops. One possibility is that high capability for suicide tends to develop during puberty, when the gap between males' and females' suicide rates grows even further (Glenn et al., 2020). Another possibility is that for some, capability for suicide develops much earlier in development. This is evidenced by work showing that even very young children (i.e., three years old) can experience suicidal thoughts and attempt suicide (Luby et al., 2019; Rosenthal \& Rosenthal, 1984). Because of this possibility, work is needed to understand what capability for suicide is across development, and whether the nature of capability for suicide changes across development, particularly for fearlessness about death.

Further work is also needed to examine what other components may comprise capability for suicide. In particular, practical capability for suicide and fearlessness about suicide seem to be promising candidates for expanding the original conceptualization of capability for suicide. In a similar vein, Joiner (2005) proposed that for those that have particularly high fearlessness about death, this fearlessness can turn into an attraction to or romanticization of death by suicide. So far, this particularly pernicious form of capability for suicide has yet to be examined, to our knowledge.

Studies that measure fearlessness about death and examine its functional neural correlates are lacking. Current evidence suggests that brain regions involved in orienting to threat, fear learning, and fear extinction may be related to fearlessness about death. Which brain regions are involved, how they develop, and how they work together to make attempting suicide feasible for those who experience suicidal ideation remains an interesting 
direction for further study. Another need is that the majority of research on capability for suicide has been done using samples from the U.S. This severely limits our ability to generalize research on the nature of capability for suicide. We call for more work to examine capability for suicide in samples that are not typically represented in this field, including from countries that are not Western, educated, industrialized, rich, and democratic (i.e., WEIRD) societies (Henrich et al., 2010). These future directions will require collaboration with scientists from around the world, including across disciplines. Our hope is that the study of capability for suicide will expand beyond clinical psychology and psychiatry, where it has received the most attention, and move to other areas such as genetics, neuroscience, and sociology. By doing so, our understanding of capability for suicide will continue to progress and, in turn, the understanding of suicidal behavior. 


\section{References}

Albanese, B. J., Macatee, R. J., Stanley, I. H., Bauer, B. W., Capron, D. W., Bernat, E., Joiner, T. E., \& Schmidt, N. B. (2019). Differentiating suicide attempts and suicidal ideation using neural markers of emotion regulation. Journal of Affective Disorders, 257, 536-550. https://doi.org/10.1016/j.jad.2019.07.014

Anestis, M. D., Bagge, C. L., Tull, M. T., \& Joiner, T. E. (2011). Clarifying the role of emotion dysregulation in the interpersonal-psychological theory of suicidal behavior in an undergraduate sample. Journal of Psychiatric Research, 45(5), 603-611. https://doi.org/10.1016/j.jpsychires.2010.10.013

Anestis, M. D., \& Capron, D. W. (2017). Deadly Experience: The Association Between Firing a Gun and Various Aspects of Suicide Risk. Suicide and Life-Threatening Behavior, 1-10. https://doi.org/10.1111/sltb.12381

Anestis, M. D., Houtsma, C., Daruwala, S. E., \& Butterworth, S. E. (2019). Firearm legislation and statewide suicide rates: The moderating role of household firearm ownership levels. Behavioral Sciences \& the Law, 37(3), 270-280. https://doi.org/10.1002/bsl.2408

Anestis, M. D., \& Joiner, T. E. (2012). Behaviorally-indexed distress tolerance and suicidality. Journal of Psychiatric Research, 46(6), 703-707. https://doi.org/10.1016/j.jpsychires.2012.02.015

Anestis, M. D., Moberg, F. B., \& Arnau, R. C. (2014). Hope and the Interpersonal-Psychological Theory of Suicidal Behavior: Replication and Extension of Prior Findings. Suicide and LifeThreatening Behavior, 44(2), 175-187. https://doi.org/10.1111/sltb.12060

Bauer, B. W., Albanese, B. J., Macatee, R. J., Tucker, R. P., Bernat, E., Schmidt, N. B., \& Capron, D. W. (2020). Fearlessness About Death is Related to Diminished Late Positive Potential Responses When Viewing Threatening and Mutilation Images in Suicidal Ideators. Cognitive Therapy and Research. https://doi.org/10.1007/s10608-020-10094-4 
Bauer, B. W., Gai, A. R., Duffy, M. E., Rogers, M. L., Khazem, L. R., Martin, R. L., Joiner, T. E., \& Capron, D. W. (2020). Fearlessness about death does not differ by suicide attempt method. Journal of Psychiatric Research, 124, 42-49. https://doi.org/10.1016/j.jpsychires.2020.02.014

Bauer, B. W., Gai, A. R., Joiner, T. E., \& Capron, D. W. (2020). The Frequency and Subjective Impact of Painful and Provocative Events on the Acquired Capability for Suicide. Archives of Suicide Research, 1-15. https://doi.org/10.1080/13811118.2020.1756017

Bauer, B. W., Martin, R. L., Allan, N. P., Fink-Miller, E. L., \& Capron, D. W. (2018). An investigation into the acquired capability for suicide. Suicide and Life-Threatening Behavior, 1-14. https://doi.org/10.1111/sltb.12502

Beck, A. T., Steer, R. A., \& Brown, G. K. (1996). Manual for the Beck Depression Inventory-II. Psychological Corporation.

Bender, T. W., Anestis, M. D., Anestis, J. C., Gordon, K. H., \& Joiner, T. E. (2012). Affective and Behavioral Paths Toward the Acquired Capacity for Suicide. Journal of Social and Clinical Psychology, 31(1), 81-100. https://doi.org/10.1521/jscp.2012.31.1.81

Bender, T. W., Gordon, K. H., Bresin, K., \& Joiner, T. E. (2011). Impulsivity and suicidality: The mediating role of painful and provocative experiences. Journal of Affective Disorders, 129, 301-307. https://doi.org/10.1016/j.jad.2010.07.023

Bill, B., Ipsch, L., Lucae, S., Pfister, H., Maragkos, M., Ising, M., \& Bronisch, T. (2012). Attempted suicide related posttraumatic stress disorder in depression-an exploratory study. Suicidology Online, 3, 138-144.

Bohns, V. K., \& Wiltermuth, S. S. (2012). It hurts when I do this (or you do that): Posture and pain tolerance. Journal of Experimental Social Psychology, 48(1), 341-345. https://doi.org/10.1016/j.jesp.2011.05.022

Boor, M., \& Bair, J. H. (1990). Suicide Rates, Handgun Control Laws, and Sociodemographic 
Variables. Psychological Reports, 66, 923-930.

Border, R., Johnson, E. C., Evans, L. M., Smolen, A., Berley, N., Sullivan, P. F., \& Keller, M. C. (2019). No Support for Historical Candidate Gene or Candidate Gene-by-Interaction Hypotheses for Major Depression Across Multiple Large Samples. American Journal of Psychiatry, 176(5), 376-387. https://doi.org/10.1176/appi.ajp.2018.18070881

Brown, G. K., Beck, A. T., Steer, R. A., \& Grisham, J. R. (2000). Risk factors for suicide in psychiatric outpatients: A 20-year prospective study. Journal of Consulting and Clinical Psychology, 68, 371-377.

Bryan, C. J., \& Rudd, M. D. (2016). The Importance of Temporal Dynamics in the Transition From Suicidal Thought to Behavior. Clinical Psychology: Science and Practice, 23(1), 21-25. https://doi.org/10.1111/cpsp.12135

Bryan, C. J., Sinclair, S., \& Heron, E. A. (2016). Do military personnel "acquire" the capability for suicide from combat? A test of the interpersonal-psychological theory of suicide. Clinical Psychological Science, 4(3), 376-385. https://doi.org/10.1177/2167702615595000

Butterworth, S. E., Daruwala, S. E., \& Anestis, M. D. (2018). Firearm storage and shooting experience: Factors relevant to the practical capability for suicide. Journal of Psychiatric Research, 102, 52-56. https://doi.org/10.1016/j.jpsychires.2018.03.010

Butterworth, S. E., Green, B. A., \& Anestis, M. D. (2017). The association between specific combat experiences and aspects of the Interpersonal Theory of Suicide. Comprehensive Psychiatry, 78, 9-18. https://doi.org/10.1016/j.comppsych.2017.06.003

Carney, D. (n.d.). My Position on "Power Poses." http://faculty.haas.berkeley.edu/dana_carney/pdf_My\%20position\%20on\%20power\%20poses .pdf

Chiurliza, B., \& Joiner, T. E. (2018). The Influence of Acetaminophen and Observational Conditioning on the Acquired Capability for Suicide. Behavior Therapy, 49(5), 681-690. 
https://doi.org/10.1016/j.beth.2017.12.009

Cho, J.-H., Deisseroth, K., \& Bolshakov, V. Y. (2013). Synaptic Encoding of Fear Extinction in mPFC-amygdala Circuits. Neuron, 80(6), 1491-1507. https://doi.org/10.1016/j.neuron.2013.09.025

Chu, C., Buchman-Schmitt, J. M., Stanley, I. H., Hom, M. A., Tucker, R. P., Hagan, C. R., Rogers, M. L., Podlogar, M. C., Chiurliza, B., Ringer, F. B., Michaels, M. S., Patros, C. H. G., \& Joiner, T. E. (2017). The interpersonal theory of suicide: A systematic review and meta-analysis of a decade of cross-national research. Psychological Bulletin, 143(12), 1313-1345. https://doi.org/10.1037/bul0000123

Clark, L. A., \& Watson, D. (2019). Constructing validity: New developments in creating objective measuring instruments. Psychological Assessment. https://doi.org/10.1037/pas0000626

Coghill, R. C., McHaffie, J. G., \& Yen, Y.-F. (2003). Neural correlates of interindividual differences in the subjective experience of pain. Proceedings of the National Academy of Sciences, 100(14), 8538-8542. https://doi.org/10.1073/pnas.1430684100

Craig, A. D. (2003). Interoception: The sense of the physiological condition of the body. Current Opinion in Neurobiology, 13(4), 500-505. https://doi.org/10.1016/S0959-4388(03)00090-4

Cronbach, L. J., \& Meehl, P. E. (1955). Construct validity in psychological tests. Psychological Bulletin, 52(4), 281-302.

Cuthbert, B. N., Schupp, H. T., Bradley, M. M., Birbaumer, N., \& Lang, P. J. (2000). Brain potentials in affective picture processing: Covariation with autonomic arousal and affective report. Biological Psychology, 52, 95-111.

Deshpande, G., Baxi, M., Witte, T., \& Robinson, J. L. (2016). A neural basis for the acquired capability for suicide. Frontiers in Psychiatry, 7, 1-19. https://doi.org/10.3389/fpsyt.2016.00125

Dhossche, D., Snell, K. S., \& Larder, S. (2000). A case-control study of tattoos in young suicide 
victims as a possible marker of risk. Journal of Affective Disorders, 59(2), 165-168. https://doi.org/10.1016/S0165-0327(99)00136-6

Duffy, M. E., Rogers, M. L., Gallyer, A. J., \& Joiner, T. E. (2019). Body Trust and Agitation: Pathways to Suicidal Thoughts and Behaviors. Archives of Suicide Research, 1-15. https://doi.org/10.1080/13811118.2019.1592039

Fazel, S., \& Runeson, B. (2020). Suicide. New England Journal of Medicine, 382(3), 266-274. https://doi.org/10.1056/NEJMra1902944

Flake, J. K., \& Fried, E. I. (2020). Measurement Schmeasurement: Questionable Measurement Practices and How to Avoid Them. Advances in Methods and Practices in Psychological Science, 3(4), 10.

Fleegler, E. W., Lee, L. K., Monuteaux, M. C., Hemenway, D., \& Mannix, R. (2013). Firearm Legislation and Firearm-Related Fatalities in the United States. JAMA Internal Medicine, 173(9), 732. https://doi.org/10.1001/jamainternmed.2013.1286

Flores, Á., Valls-Comamala, V., Costa, G., Saravia, R., Maldonado, R., \& Berrendero, F. (2014). The Hypocretin/Orexin System Mediates the Extinction of Fear Memories.

Neuropsychopharmacology, 39(12), 2732-2741. https://doi.org/10.1038/npp.2014.146

Franklin, J. C., Fox, K. R., Franklin, C. R., Kleiman, E. M., Ribeiro, J. D., Jaroszewski, A. C., Hooley, J. M., \& Nock, M. K. (2016). A brief mobile app reduces nonsuicidal and suicidal self-injury: Evidence from three randomized controlled trials. Journal of Consulting and Clinical Psychology, 84(6), 544-557.

Franklin, J. C., Hessel, E. T., \& Prinstein, M. J. (2011). Clarifying the role of pain tolerance in suicidal capability. Psychiatry Research, 189(3), 362-367. https://doi.org/10.1016/j.psychres.2011.08.001

Franklin, J. C., Ribeiro, J. D., Fox, K. R., Bentley, K. H., Kleiman, E. M., Huang, X., Musacchio, K. M., Jaroszewski, A. C., Chang, B. P., \& Nock, M. K. (2017). Risk factors for suicidal 
thoughts and behaviors: A meta-analysis of 50 years of research. Psychological Bulletin, 143(2), 187-232. https://doi.org/10.1037/bul0000084

Gauthier, J. M., Hollingsworth, D. W., \& Bagge, C. L. (2018). Number and violence of suicide attempt methods: A preliminary investigation of the associations with fearlessness of suicide and fearlessness about death. Psychiatry Research, 265(March), 183-189. https://doi.org/10.1016/j.psychres.2018.04.050

Gauthier, J. M., Zuromski, K. L., Gitter, S. A., Witte, T. K., Cero, I. J., Gordon, K. H., Ribeiro, J., Anestis, M., \& Joiner, T. (2014). The interpersonal-psychological theory of suicide and exposure to video game violence. Journal of Social and Clinical Psychology, 33(6), 512-535. https://doi.org/10.1521/jscp.2014.33.6.512

Glenn, C. R., Kleiman, E. M., Kellerman, J., Pollak, O., Cha, C. B., Esposito, E. C., Porter, A. C., Wyman, P. A., \& Boatman, A. E. (2020). Annual Research Review: A meta-analytic review of worldwide suicide rates in adolescents. Journal of Child Psychology and Psychiatry, 61(3), 294-308. https://doi.org/10.1111/jcpp.13106

Goldberg, S. B., Tucker, R. P., Abbas, M., Schultz, M. E., Hiserodt, M., Thomas, K. A., Anestis, M. D., \& Wyman, M. F. (2019). Firearm Ownership and Capability for Suicide in Post-Deployment National Guard Service Members. Suicide and Life-Threatening Behavior, 49(6), 1668-1679. https://doi.org/10.1111/sltb.12551

Gould, T. D., Georgiou, P., Brenner, L. A., Brundin, L., Can, A., Courtet, P., Donaldson, Z. R., Dwivedi, Y., Guillaume, S., Gottesman, I. I., Kanekar, S., Lowry, C. A., Renshaw, P. F., Rujescu, D., Smith, E. G., Turecki, G., Zanos, P., Zarate, C. A., Zunszain, P. A., \& Postolache, T. T. (2017). Animal models to improve our understanding and treatment of suicidal behavior. Translational Psychiatry, 7(4), e1092-e1092. https://doi.org/10.1038/tp.2017.50

Granato, S. L., Smith, P. N., \& Selwyn, C. N. (2015). Acquired capability and masculine gender norm adherence: Potential pathways to higher rates of male suicide. Psychology of Men \& 
Masculinity, 16(3), 246-253. https://doi.org/10.1037/a0038181

Grasso, D. J., \& Simons, R. F. (2011). Perceived parental support predicts enhanced late positive event-related brain potentials to parent faces. Biological Psychology, 86, 26-30. https://doi.org/10.1016/j.biopsycho.2010.10.002

Gutierrez, P. M., Pease, J., Matarazzo, B. B., Monteith, L. L., Hernandez, T., \& Osman, A. (2016). Evaluating the psychometric properties of the Interpersonal Needs Questionnaire and the Acquired Capability for Suicide Scale in military veterans. Psychological Assessment, 28(12), 1684-1694. https://doi.org/10.1037/pas0000310

Hajcak, G., \& Foti, D. (2020). Significance?... Significance! Empirical, methodological, and theoretical connections between the late positive potential and P300 as neural responses to stimulus significance: An integrative review. Psychophysiology, e13570. https://doi.org/10.1111/psyp.13570

Hajcak, G., Klawohn, J., \& Meyer, A. (2019). The Utility of Event-Related Potentials in Clinical Psychology. Annual Review of Clinical Psychology, 15(1), 71-95. https://doi.org/10.1146/annurev-clinpsy-050718-095457

Hallensleben, N., Glaesmer, H., Forkmann, T., Rath, D., Strauss, M., Kersting, A., \& Spangenberg, L. (2019). Predicting suicidal ideation by interpersonal variables, hopelessness and depression in real-time. An ecological momentary assessment study in psychiatric inpatients with depression. European Psychiatry, 56(1), 43-50. https://doi.org/10.1016/j.eurpsy.2018.11.003

Hallensleben, N., Spangenberg, L., Forkmann, T., Rath, D., Hegerl, U., Kersting, A., Kallert, T. W., \& Glaesmer, H. (2018). Investigating the Dynamics of Suicidal Ideation: Preliminary Findings From a Study Using Ecological Momentary Assessments in Psychiatric Inpatients. Crisis, 39(1), 65-69. https://doi.org/10.1027/0227-5910/a000464

Hamaker, E. L., Kuiper, R. M., \& Grasman, R. P. P. P. (2015). A critique of the cross-lagged panel model. Psychological Methods, 20(1), 102-116. https://doi.org/10.1037/a0038889 
Hames, J. L., Rogers, M. L., Silva, C., Ribeiro, J. D., Teale, N. E., \& Joiner, T. E. (2017). A Social Exclusion Manipulation Interacts with Acquired Capability for Suicide to Predict SelfAggressive Behaviors. Archives of Suicide Research. https://doi.org/10.1080/13811118.2017.1304309

Harden, K. P., \& Koellinger, P. D. (2020). Using genetics for social science. Nature Human Behaviour, 4(6), 567-576. https://doi.org/10.1038/s41562-020-0862-5

Hawkins, K. A., Hames, J. L., Ribeiro, J. D., Silva, C., Joiner, T. E., \& Cougle, J. R. (2014). An examination of the relationship between anger and suicide risk through the lens of the interpersonal theory of suicide. Journal of Psychiatric Research, 50, 59-65. https://doi.org/10.1016/j.jpsychires.2013.12.005

Henrich, J., Heine, S. J., \& Norenzayan, A. (2010). The Weirdest People in the World. 71.

Joiner, T. E. (2005). Why people die by suicide. Harvard University Press.

Joiner, T. E., Conwell, Y., Fitzpatrick, K. K., Witte, T. K., Schmidt, N. B., Berlim, M. T., Fleck, M. P. A., \& Rudd, M. D. (2005). Four studies on how past and current suicidality relate even when "everything but the kitchen sink" is covaried. Journal of Abnormal Psychology, 114, 291-303. https://doi.org/10.1037/0021-843X.114.2.291

Khazem, L. R., Houtsma, C., Gratz, K. L., Tull, M. T., Green, B. A., \& Anestis, M. D. (2015). Firearms Matter: The Moderating Role of Firearm Storage in the Association Between Current Suicidal Ideation and Likelihood of Future Suicide Attempts Among United States Military Personnel. Military Psychology, 28(1), 25-33. https://doi.org/10.1037/mil0000099

Klawohn, J., Burani, K., Bruchnak, A., Santopetro, N., \& Hajcak, G. (2020). Reduced neural response to reward and pleasant pictures independently relate to depression. Psychological Medicine, 1-9. https://doi.org/10.1017/S0033291719003659

Kleiman, E. M., Turner, B. J., Fedor, S., Beale, E. E., Huffman, J. C., \& Nock, M. K. (2017). Examination of real-time fluctuations in suicidal ideation and its risk factors: Results from 
two ecological momentary assessment studies. Journal of Abnormal Psychology, 126(6), 726-738. https://doi.org/10.1037/abn0000273

Klimek, V., Stockmeier, C., Overholser, J., Meltzer, H. Y., Kalka, S., Dilley, G., \& Ordway, G. A. (1997). Reduced Levels of Norepinephrine Transporters in the Locus Coeruleus in Major Depression. The Journal of Neuroscience, 17(21), 8451-8458.

https://doi.org/10.1523/JNEUROSCI.17-21-08451.1997

Klonsky, E. D., \& May, A. M. (2015). The Three-Step Theory (3ST): A new theory of suicide in the “ideation-to-action” framework. International Journal of Cognitive Therapy, 8(2), 114-129.

Koenig, J., Rinnewitz, L., Niederbäumer, M., Strozyk, T., Parzer, P., Resch, F., \& Kaess, M. (2017). Longitudinal development of pain sensitivity in adolescent non-suicidal self-injury. Journal of Psychiatric Research, 89, 81-84. https://doi.org/10.1016/j.jpsychires.2017.02.001

Lakens, D. (2017). Equivalence Tests: A Practical Primer for $t$ Tests, Correlations, and MetaAnalyses. Social Psychological and Personality Science, 8(4), 355-362. https://doi.org/10.1177/1948550617697177

Liu, Y., Huang, H., Mcginnis-Deweese, M., Keil, A., \& Ding, M. (2012). Neural substrate of the late positive potential in emotional processing. Journal of Neuroscience, 32(42), 14563-14572. https://doi.org/10.1523/JNEUROSCI.3109-12.2012

Longwell, B. T., \& Truax, P. (2005). The differential effects of weekly, monthly, and bimonthly administrations of the beck Depression Inventory-II: Psychometric properties and clinical implications. Behavior Therapy, 36(3), 265-275. https://doi.org/10.1016/S00057894(05)80075-9

Luby, J. L., Whalen, D., Tillman, R., \& Barch, D. M. (2019). Clinical and Psychosocial Characteristics of Young Children With Suicidal Ideation, Behaviors, and Nonsuicidal SelfInjurious Behaviors. Journal of the American Academy of Child \& Adolescent Psychiatry, 58(1), 117-127. https://doi.org/10.1016/j.jaac.2018.06.031 
Luck, S. J. (2014). An introduction to the event-related potential technique (2nd ed.). The MIT Press. Ludwig, J., \& Cook, P. J. (2000). Homicide and Suicide Rates Associated With Implementation of the Brady Handgun Violence Prevention Act. JAMA, 284(5), 585. https://doi.org/10.1001/jama.284.5.585

Mehling, W. E., Price, C., Daubenmier, J. J., Acree, M., Bartmess, E., \& Stewart, A. (2012). The Multidimensional Assessment of Interoceptive Awareness (MAIA). PLoS ONE, 7(11). https://doi.org/10.1371/journal.pone.0048230

Mitchell, S. M., Cukrowicz, K. C., Van Allen, J., \& Seegan, P. L. (2015). Moderating Role of Trait Hope in the Relation Between Painful and Provocative Events and Acquired Capability for Suicide. Crisis, 36(4), 249-256. https://doi.org/10.1027/0227-5910/a000319

Mitchell, S. M., Jahn, D. R., \& Cukrowicz, K. C. (2014). The Relation Between Illegal Risk Behaviors and the Acquired Capability for Suicide. Crisis, 35(6), 368-377. https://doi.org/10.1027/0227-5910/a000274

Mitchell, S. M., Jahn, D. R., Guidry, E. T., \& Cukrowicz, K. C. (2015). The Relationship Between Video Game Play and the Acquired Capability for Suicide: An Examination of Differences by Category of Video Game and Gender. Cyberpsychology, Behavior, and Social Networking, 18(12), 757-762. https://doi.org/10.1089/cyber.2015.0171

Naghavi, M. (2019). Global, regional, and national burden of suicide mortality 1990 to 2016: Systematic analysis for the Global Burden of Disease Study 2016. BMJ, 194. https://doi.org/10.1136/bmj.194

Nieuwenhuis, S., De Geus, E. J., \& Aston-Jones, G. (2011). The anatomical and functional relationship between the $\mathrm{P} 3$ and autonomic components of the orienting response: $\mathrm{P} 3$ and orienting response. Psychophysiology, 48(2), 162-175. https://doi.org/10.1111/j.14698986.2010.01057.x

Öhman, A. (2005). The role of the amygdala in human fear: Automatic detection of threat. 
Psychoneuroendocrinology, 30(10), 953-958. https://doi.org/10.1016/j.psyneuen.2005.03.019

Orbach, I. (1996). The role of the body experience in self-destruction. Clinical Child Psychology and Psychiatry, 1(4), 607-619.

Ribeiro, J. D., Harris, L. M., Linthicum, K. P., \& Bryen, C. P. (2020). Do Suicidal Behaviors Increase the Capability for Suicide? A Longitudinal Pretest-Posttest Study of More Than 1,000 HighRisk Individuals. Clinical Psychological Science, 216770262092151. https://doi.org/10.1177/2167702620921511

Ribeiro, J. D., Witte, T. K., Van Orden, K. A., Selby, E. A., Gordon, K. H., Bender, T. W., \& Joiner, T. E. (2014). Fearlessness about death: The psychometric properties and construct validity of the revision to the Acquired Capability for Suicide Scale. Psychological Assessment, 26(1), 115126. https://doi.org/10.1037/a0034858

Rogers, M. L., Bauer, B. W., Gai, A. R., Duffy, M. E., \& Joiner, T. E. (2021). Examination of measurement invariance of the Acquired Capability for Suicide Scale. Psychological Assessment. https://doi.org/10.1037/pas0000998

Rogers, M. L., Hagan, C. R., \& Joiner, T. E. (2018). Examination of interoception along the suicidality continuum. Journal of Clinical Psychology.

Rogers, M. L., \& Joiner, T. E. (2019). Exploring the temporal dynamics of the interpersonal theory of suicide constructs: A dynamic systems modeling approach. Journal of Consulting and Clinical Psychology, 87(1), 56-66. https://doi.org/10.1037/ccp0000373

Rosenthal, P. A., \& Rosenthal, S. (1984). Suicidal behavior by preschool children. American Journal of Psychiatry, April, 520-525.

Rosenthal, R. J., Rinzler, C., Wallsh, R., \& Klausner, E. (1972). Wrist-Cutting Syndrome: The Meaning of a Gesture. American Journal of Psychiatry, 128(11), 1363-1368. https://doi.org/10.1176/ajp.128.11.1363

Sara, S. J., \& Bouret, S. (2012). Orienting and Reorienting: The Locus Coeruleus Mediates 
Cognition through Arousal. Neuron, 76(1), 130-141.

https://doi.org/10.1016/j.neuron.2012.09.011

Schernhammer, E. S., \& Colditz, G. A. (2004). Suicide Rates Among Physicians: A Quantitative and Gender Assessment (Meta-Analysis). Am J Psychiatry, 8.

Sears, R. M., Fink, A. E., Wigestrand, M. B., Farb, C. R., de Lecea, L., \& LeDoux, J. E. (2013). Orexin/hypocretin system modulates amygdala-dependent threat learning through the locus coeruleus. Proceedings of the National Academy of Sciences, 110(50), 20260-20265. https://doi.org/10.1073/pnas.1320325110

Shahnaz, A., Bauer, B. W., Daruwala, S. E., \& Klonsky, E. D. (2020). Exploring the scope and structure of suicide capability. Suicide and Life-Threatening Behavior, 50(6), 1230-1240. https://doi.org/10.1111/sltb.12686

Shiffman, S., Stone, A. A., \& Hufford, M. R. (2008). Ecological momentary assessment. Annual Review of Clinical Psychology, 4, 1-32.

https://doi.org/10.1146/annurev.clinpsy.3.022806.091415

Smith, A. R., Ribeiro, J. D., Mikolajewski, A., Taylor, J., Joiner, T. E., \& Iacono, W. G. (2012). An examination of environmental and genetic contributions to the determinants of suicidal behavior among male twins. Psychiatry Research, 197, 60-65. https://doi.org/10.1016/j.psychres.2012.01.010

Smith, P. N., Wolford-Clevenger, C., Mandracchia, J. T., \& Jahn, D. R. (2013). An exploratory factor analysis of the Acquired Capability for Suicide Scale in male prison inmates. Psychological Services, 10(1), 97.

Soloff, P. H., Lis, J. A., Kelly, T., Cornelius, J., \& Ulrich, R. (1994). Risk factors for suicidal behavior in borderline personality disorder. American Journal of Psychiatry, 151(9), 13161323. https://doi.org/10.1176/ajp.151.9.1316

Soloff, P. H., Lynch, K. G., Kelly, T. M., Malone, K. M., \& Mann, J. J. (2000). Characteristics of 
Suicide Attempts of Patients With Major Depressive Episode and Borderline Personality

Disorder: A Comparative Study. American Journal of Psychiatry, 157(4), 601-608.

https://doi.org/10.1176/appi.ajp.157.4.601

Solomon, R. L., \& Corbit, J. D. (1974). An opponent-process theory of motivation: I. Temporal dynamics of affect. Psychological Review, 81(2), 119-145. https://doi.org/10.1037/h0036128

Spangenberg, L., Glaesmer, H., Hallensleben, N., Rath, D., \& Forkmann, T. (2019). (In)stability of Capability for Suicide in Psychiatric Inpatients: Longitudinal Assessment Using Ecological Momentary Assessments. Suicide and Life-Threatening Behavior. https://doi.org/10.1111/sltb.12547

Stanley, I. H., Boffa, J. W., \& Joiner, T. E. (2018). PTSD from a suicide attempt: Phenomenological and diagnostic considerations. Psychiatry: Interpersonal and Biological Processes. https://doi.org/10.1080/00332747.2018.1485373

Stanley, I. H., Hom, M. A., Boffa, J. W., Stage, D. L., \& Joiner, T. E. (2019). PTSD from a suicide attempt: An empirical investigation among suicide attempt survivors. Journal of Clinical Psychology, 75(10), 1879-1895. https://doi.org/10.1002/jclp.22833

Stanley, I. H., Hom, M. A., Gallyer, A. J., Gray, J. S., \& Joiner, T. E. (2019). Suicidal behaviors among American Indian/Alaska Native firefighters: Evidence for the role of painful and provocative events. Transcultural Psychiatry, 136346151984781.

https://doi.org/10.1177/1363461519847812

Starr, C. J., Sawaki, L., Wittenberg, G. F., Burdette, J. H., Oshiro, Y., Quevedo, A. S., \& Coghill, R. C. (2009). Roles of the Insular Cortex in the Modulation of Pain: Insights from Brain Lesions. Journal of Neuroscience, 29(9), 2684-2694. https://doi.org/10.1523/JNEUROSCI.517308.2009

Stone, A. A., \& Shiffman, S. (1994). Ecological Momentary Assessment (Ema) in Behavioral Medicine. Annals of Behavioral Medicine, 16(3), 199-202. 
https://doi.org/10.1093/abm/16.3.199

Teismann, T., Forkmann, T., Wachtel, S., Edel, M.-A., Nyhuis, P., \& Glaesmer, H. (2015). The

German version of the Painful and Provocative Events Scale: A psychometric investigation.

Psychiatry Research, 226(1), 264-272. https://doi.org/10.1016/j.psychres.2014.12.060

Teismann, T., Förtsch, E.-M. A. D., Baumgart, P., Het, S., \& Michalak, J. (2014). Influence of violent video gaming on determinants of the acquired capability for suicide. Psychiatry Research, 215(1), 217-222. https://doi.org/10.1016/j.psychres.2013.10.021

Van Orden, K. A., Witte, T. K., Cukrowicz, K. C., Braithwaite, S. R., Selby, E. A., \& Joiner, T. E. (2010). The interpersonal theory of suicide. Psychological Review, 117(2), 575-600. https://doi.org/10.1037/a0018697

Van Orden, K. A., Witte, T. K., Gordon, K. H., Bender, T. W., \& Joiner, T. E. (2008). Suicidal desire and the capability for suicide: Tests of the interpersonal-psychological theory of suicidal behavior among adults. Journal of Consulting and Clinical Psychology, 76(1), 72-83. https://doi.org/10.1037/0022-006X.76.1.72

Velkoff, E. A., \& Smith, A. R. (2019). Examining Patterns of Change in the Acquired Capability for Suicide Among Eating Disorder Patients. Suicide and Life-Threatening Behavior, 49(4), 1032-1043. https://doi.org/10.1111/sltb.12505

Wachtel, S., Vocks, S., Edel, M.-A., Nyhuis, P., Willutzki, U., \& Teismann, T. (2014). Validation and psychometric properties of the German Capability for Suicide Questionnaire. Comprehensive Psychiatry, 55(5), 1292-1302. https://doi.org/10.1016/j.comppsych.2014.03.008

Weinberg, A., \& Hajcak, G. (2010). Beyond good and evil: The time-course of neural activity elicited by specific picture content. Emotion, 10(6), 767-782. https://doi.org/10.1037/a0020242

Weinberg, A., \& Hajcak, G. (2011). The late positive potential predicts subsequent interference with target processing. Journal of Cognitive Neuroscience, 23(10), 2994-3007. 
http://dx.doi.org.proxy.lib.fsu.edu/10.1162/jocn.2011.21630

Weinberg, A., May, A. M., Klonsky, E. D., Kotov, R., \& Hajcak Greg. (2017). Decreased neural response to threat differentiates patients who have attempted suicide from nonattempters with current ideation. Clinical Psychological Science. https://doi.org/10.1177/2167702617718193

Weinberg, A., Perlman, G., Kotov, R., \& Hajcak, G. (2016). Depression and reduced neural response to emotional images: Distinction from anxiety, and importance of symptom dimensions and age of onset. Journal of Abnormal Psychology, 125(1), 26-39.

https://doi.org/10.1037/abn0000118

Willoughby, T., Heffer, T., \& Hamza, C. A. (2015). The link between nonsuicidal self-injury and acquired capability for suicide: A longitudinal study. Journal of Abnormal Psychology, 124(4), 1110-1115. https://doi.org/10.1037/abn0000104

World Health Organization. (2014). Preventing suicide: A global imperative. World Health Organization. https://doi.org/10.1002/9780470774120

Yang, L., Pan, D., Li, Z., \& Liu, X. (2019). Validation of a Chinese Version of the Acquired Capability for Suicide Scale. Death Studies, 1-8. https://doi.org/10.1080/07481187.2019.1671545

Zuromski, K. L., Cero, I., \& Witte, T. K. (2018). Non-monotonic temporal variation in fearlessness about death: A latent class growth analysis. Psychiatry Research, 268, 46-52. https://doi.org/10.1016/j.psychres.2018.06.057 